Vol. 6, No. 3-4, 2020

Volodymyr Gurey

Department of Mechanics and Automation Engineering, Lviv Polytechnic National University, Ukraine, Lviv, S. Bandery Street 12, E-mail: Volodymyr.I.Hurei@1pnu.ua, ORCID 0000-0002-5117-6802

\title{
TOPOGRAPHY OF THE STRENGTHENED CYLINDRICAL SURFACE AFTER FRICTIONAL CONTINUOUS TREATMENT
}

Received: August 19, 2020 / Revised: September 30, 2020 / Accepted: December 30, 2020

(C) Gurey V., 2020

\begin{abstract}
Friction treatment refers to methods of surface strengthening (hardening) of the parts' working surfaces using highly concentrated energy sources. Concentrated energy flow is formed during high-speed friction of the tool on the treated surface in the area of their contact. A strengthened (reinforced) white layer with a nanocrystalline structure is formed in the surface layer of the treated surface. Friction treatment of cylindrical surfaces of samples made of steel $41 \mathrm{Cr} 4$ (hardening and low-temperature tempering) was made on a lathe, and the device for the autonomous drive of the strengthening tool was installed instead of a toolpost. The tool was used with a smooth working part and with transverse grooves on the working part. Experimental researches of the strengthened surfaces' topography were carried out on a profilometer "TALYScan 150" (Taylor Hobson Ltd, UK). The obtained data were processed in the software "Digital Surf MountainsLab Premium 8.2". After friction treatment by using the tool with transverse grooves on its working part on the treated surface more evenly distributed peaks than after friction treatment by using the tool with the smooth surface. Analysing the spectral density of the peak's distribution on the treated surfaces, it can be noted that after friction treatment by using the tool with transverse grooves, the area of the spectra is the lowest in comparison with friction treatment by using the tool with a smooth working part. When using the tool with cross grooves on its working part during frictional treatment allows to receive the best parameters of quality of the treatment surface in comparison with frictional treatment by the tool with a smooth working part. The parameters of the load-bearing capacity curve of the surface treated by the tool with transverse grooves on its working surface are better than after frictional treatment by the tool with a smooth working part. The treated surface by the tool with transverse grooves has a more favourable surface for wear, which was confirmed by research on wear resistance.
\end{abstract}

Keywords: white layer, wear, nanocrystalline structure, friction treatment, bearing curve, roughness, waviness.

\section{Introduction}

The service properties of parts depend on the quality parameters of the contact surfaces and the surface layer, which are determined by geometric (macrodeviations, waviness, roughness), physical, and mechanical (microhardness, residual stresses, structure) characteristics and mutual location of microroughness on the contact surfaces. The specified parameters depend on the surface treatment technology of parts and assembly of products $[1,2]$.

The contact interaction of the parts' surfaces determines the actual contact area, contact stiffness, the magnitude of local stresses, the conditions of the oil film formation, and other indicators that affect machine parts efficiency [3, 4]. The contact interaction is significantly influenced by the microgeometry and physical and mechanical surface characteristics, such as the actual contact area, which depends on the shape and size of peaks and their distribution (bearing capacity curve), maximum pressure and contact nature $[5,6]$. 


\section{Volodymyr Gurey}

\section{Methodology}

Surface methods of strengthening (hardening) that using highly concentrated energy sources are used in mechanical engineering. These are laser, plasma, electron-beam, and other treatments [7, 8]. In these methods, the high-intensity concentrated energy flows for a short time and locally act at high speeds on relatively small volumes of the metal surface layer. A high-speed cooling of the heated metal occurs after the removal of the energy source [9, 10]. Friction treatment refers to surface strengthening (hardening) methods using highly concentrated energy sources. During this treatment in the surface layer of the parts is occurring intense shear deformation of the metal. Structural and phase transformations with the formation of the strengthened (reinforced) layers with nanocrystalline structures, which have specific properties take place in the surface layers of parts [11].

The conducted researches of the strengthened surfaces were mostly based on the determination of roughness parameters on height and pitch. The aim of this work is to determine the influence of friction treatment parameters on the stereometric characteristics of the treated surfaces.

In the process of friction treatment, a highly concentrated flow of energy is formed in the contact area of the tool-part during high-speed friction of the tool on the treated surface of the part. At the same time, there is a simultaneous high-speed shear deformation of the metal surface layer. The heating rate reaches $10^{5}-10^{6} \mathrm{~K} / \mathrm{s}$. The metal surface layers are heated to temperatures above the point of phase transformations $\left(\mathrm{A}_{\mathrm{c} 3}\right)$. The heat is removing to the depth of the metal and the cooling rate is $10^{4}-5 \cdot 10^{5} \mathrm{~K} / \mathrm{s}$. The strengthened white layer with a nanocrystalline structure is formed in the surface layer of machine parts [12].

Cylindrical samples were made of steel $41 \mathrm{Cr} 4$ (hardening and low-temperature tempering). Friction treatment of samples' cylindrical surfaces was performed on an upgraded lather machine (model 16K20, USSR), and the device for the autonomous drive of the strengthening tool was installed instead of a toolpost.

Experimental researches of the strengthened surfaces' topography were carried out on a profilometer "TALYScan 150" (Taylor Hobson Ltd, UK) (Fig. 1). The zone of samples' treated surfaces with the size of $6 \times 6 \mathrm{~mm}$ was scanned by using a diamond needle with a radius at the top that had $0.2 \mu \mathrm{m}$. The speed of the needle was $3000 \mathrm{~mm} / \mathrm{min}$, the pitch was $5 \mu \mathrm{m}$. Processing of the obtained results was carried out using the software "Digital Surf MountainsLab Premium 8.2". The software gives the possibility to expand the scanned curved surface into a flat one. The 3D view of the surface; particle analysis; peak count distribution; volume of a hole or a peak; frequency spectrum; average power spectrum density; material ratio curve; $S k$ parameters were obtained by using this software [13].

Tools with a smooth working surface are mainly used for friction strengthening (hardening). To increase the intensity of shear deformation of the treated surface layer, the tool was made with transverse grooves on its working surface. The groove width was 3-4 $\mathrm{mm}$ and was selected on the condition of the guaranteed complete outlet from the contact of the tool with the part. The grooves number was chosen from the condition of their even number. To improve the quality parameters of the part's treated surface and eliminate setting between the part's treated surface and working surfaces of the tool during friction treatment the technological medium is applied. Also, the technological medium in the process of treatment under the action of high temperatures and pressures decomposes and is the source for the diffusion of its components into the surface layers of the treated material. Mineral oil is mainly used, which in the treatment process decomposes into atomic carbon and other elements then diffuse them into the surface layers of the sample, and promote the formation of the high-quality strengthened layers. In our case, as a technological medium used mineral oil with active additives containing polymers. To carry the technological medium to the treating area was used the standard cooling system of the machine [14].

When the groove passes over the contact area, the tool loss the contact with the treated surface and due the action of elastic deformations, the volume of the contact zone increases. When coming into contact with the next tool's smooth working surface with the treated part, the shock load of the contact zone takes place. 


\section{Topography of the Strengthened Cylindrical Surface after Frictional Continuous Treatment}

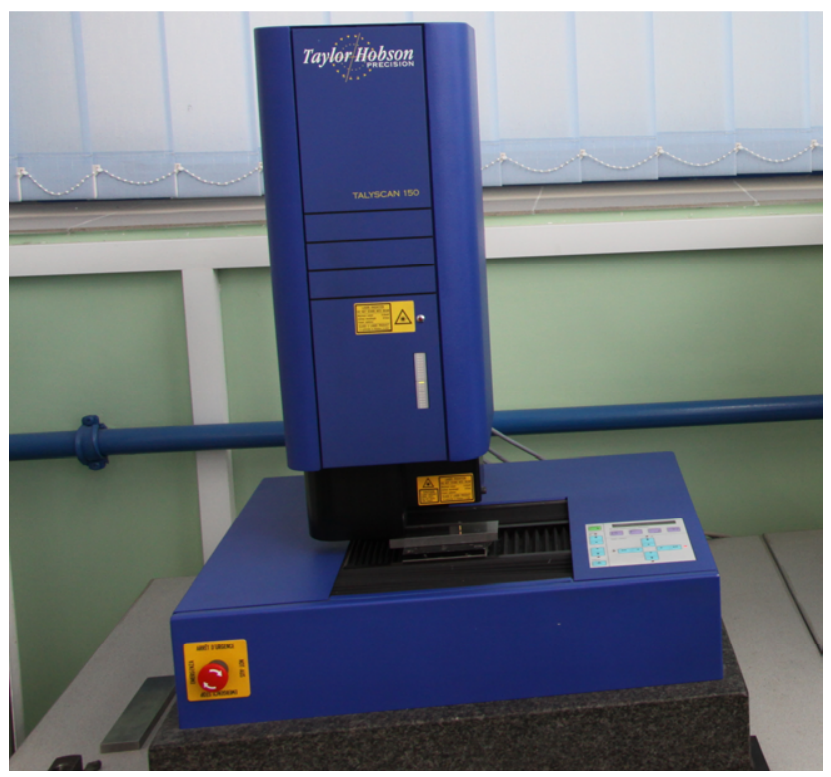

Fig. 1. Profilometer "TALYScan 150" (Taylor Hobson Ltd, UK)

To compare the way how the shape of the tool working surface affects the interaction force components in the contact area of the tool-part during friction treating were used the tools with a smooth working part and with transverse grooves.

\section{Results and Discussion}

Frictional strengthening of cylindrical surfaces, according to the kinematics of the process, is similar to the process of circular grinding. To determine the normal $P_{y}$ and tangential $P_{z}$ components of the interaction force that occurs in the tool-part contact area, the specially designed dynamometer was used, on which the two force components were registered simultaneously. The signals from the dynamometer were transmit to an analog-to-digital converter (frequency $100 \mathrm{kHz}$ ). The research data processing was by using the software "PowerGraph" (Fig. 2).

Researches have shown that the shape of the working part of the tool significantly affects into the components of the interaction force during friction treating. The largest values of the interaction force components $P_{y}, P_{z}$ are obtained when treating by the tool with a smooth working part. Using the tool with transverse grooves leads to a decrease the interaction force components.

Thus, when using a tool with a smooth working part, the normal component $P_{y}$ of the interaction force is equal $1280 \mathrm{~N}$, and tangential $P_{z}$ is equal $76 \mathrm{~N}$. When using the tool with transverse grooves on the working part, the normal component $P_{y}$ of the interaction force is equal $1060 \mathrm{~N}$, and the tangential $P_{z}$ is equal $58 \mathrm{~N}$. The decreasing of the components $P_{y}, P_{z}$ of the interaction force is $17 \%$ and $23 \%$, respectively, in comparison with the tool that have a smooth working part.

Using the tool with transverse grooves leads to decrease the interaction force components in the contact zone. This significantly affects into the loading of the machine's spindle assembly, and also reduces the power-intensive of the surface strengthening process of machine parts during friction treating by the tool with a smooth working part. It should be noted that during the friction treatment by using the tool with transverse grooves, the reinforced layer of increased thickness is obtained and the quality parameters of the treated surface are improved.

The obtained data of the interaction force components in the contact area of the tool-part show that the components of the interaction force have a wave character, which is the same according to the phase. The oscillation amplitude of the interaction force components reaches to $0.3-0.4$ of the force that occurs in the contact area of the tool-part, and the oscillation period is $20-22 \mathrm{~ms}$. The oscillation period less depends on the treating modes. Oscillations of the interaction force components in the contact area of the tool-part occur due to the fact that a single contact is heated to high temperatures close to the melting point, then occurs the softening of the treated surface and the coefficient of friction reduces (see Fig. 2). 

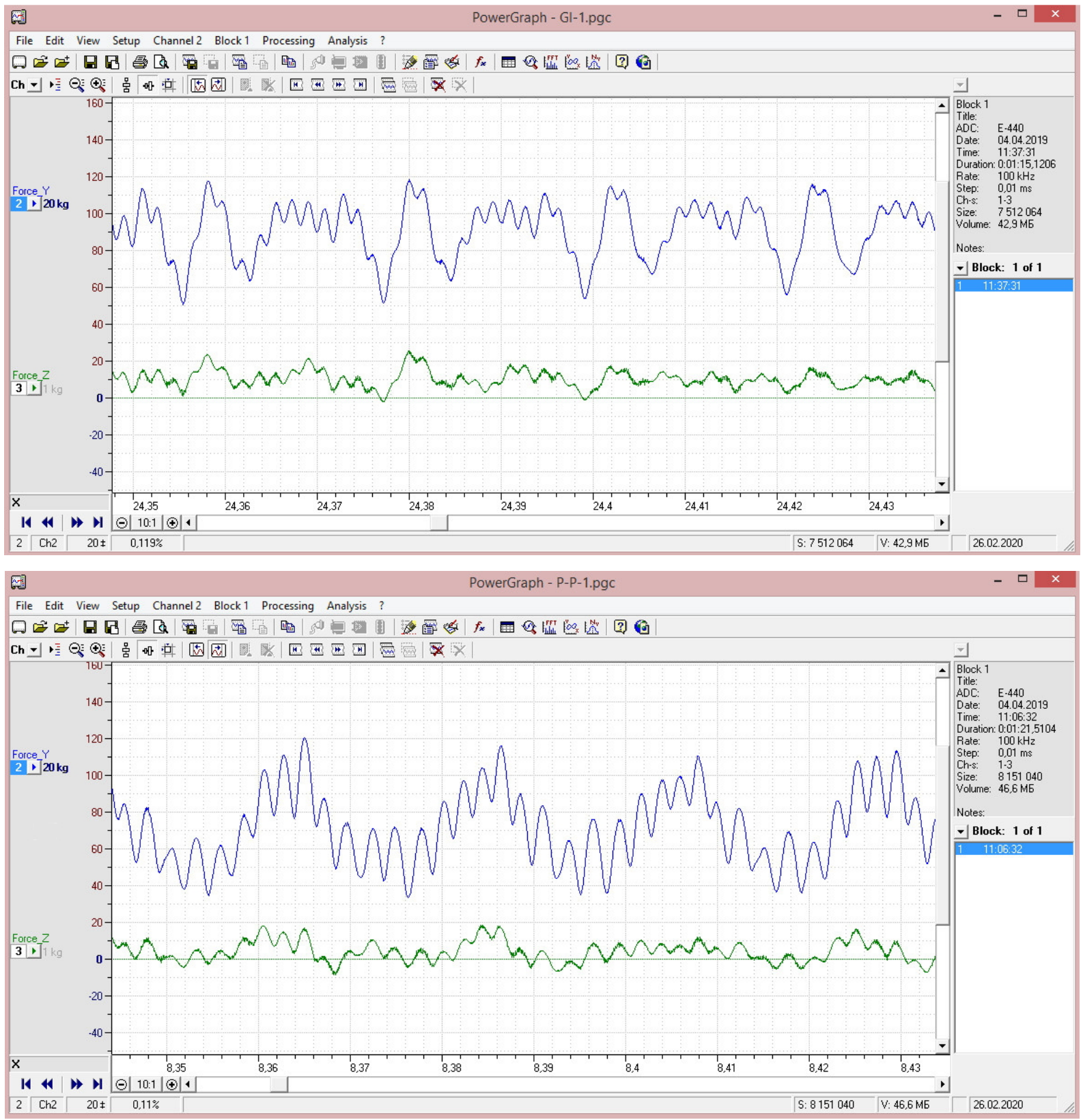

Fig. 2. Fragment record of the interaction force components that occurs in the contact area of the tool-part due the friction treatment by the tool with a smooth (a) and with a continuous working surfaces (b)

The formation of surface microgeometry and surface layer quality parameters is a complex physical process with active physicochemical interaction of all materials (materials of tool and treated part and technological medium) in the treatment area. In this case, the treated surface is the result of copying the displacement trajectories of the tool's surface relative to the part, and which form a macro-and microprofile. The constant roughness of the treated surface depends on the geometric parameters and vibrations of the machine-tool-equipment-part system and it is formed after several passes of the tool's working surface on some zone of the part (workpiece).

Not only the roughness of the treated surface is very important, but also its waviness which is a combination of periodic and aperiodic peaks and pits. The oscillations of the treated part, the spindle assembly, the strengthening tool, and the shape of its working surface and radial beating have the most actively influenced into formation the waviness of the treated surface during finishing operation. The ratio of part speeds and the tool, their sizes, number of passes, and waves phases shift at the following passes also have a significant meaning. 


\section{Topography of the Strengthened Cylindrical Surface after Frictional Continuous Treatment}

Researches have shown that the modes of friction treating, the shape of the tool's working surface, and other parameters are significantly influenced into the parameters of the stereometry of the treated part's surface.

Analysing the topography of the surface after friction treatment, it can be noted that on the treated surface clearly visible traces of the transverse feed of the tool. When the maximum temperature is close to the melting point, on the treated surface in the area of single contact of the tool-part the strength of the material decreases and under the action of the clamping force the metal is pushed away from the contact area.

When using the tool with a smooth working surface, on the part's treated surface the shear deformation occurs only in the direction of the tool rotation. The formation parameters of the treated surface quality occur due to unidirectional friction of the tool on the contact surface of the part. When using the tool with transverse grooves on the working surface, in addition to shear deformation the shock load takes place in the tool single area additionally passes at the moment when groove contacting with the treated surface with a frequency near $2 \mathrm{kHz}$.

Analysis of the topography of the treated surface by friction treatment using the tool with a smooth working surface showed that the height of the distortion of the deformed surface is up to $10 \mu \mathrm{m}$ (Fig. 3). The waviness of the surface after friction treatment is different in the longitudinal and transverse directions. In the transverse direction before friction treatment, the height of the waviness is about $7 \mu \mathrm{m}$ and the pitch is about $2 \mathrm{~mm}$, but in the longitudinal direction the waviness is not clearly detected. The height of the profile is also about $9 \mu \mathrm{m}$ but the pitch is indefinable (Fig. 4).

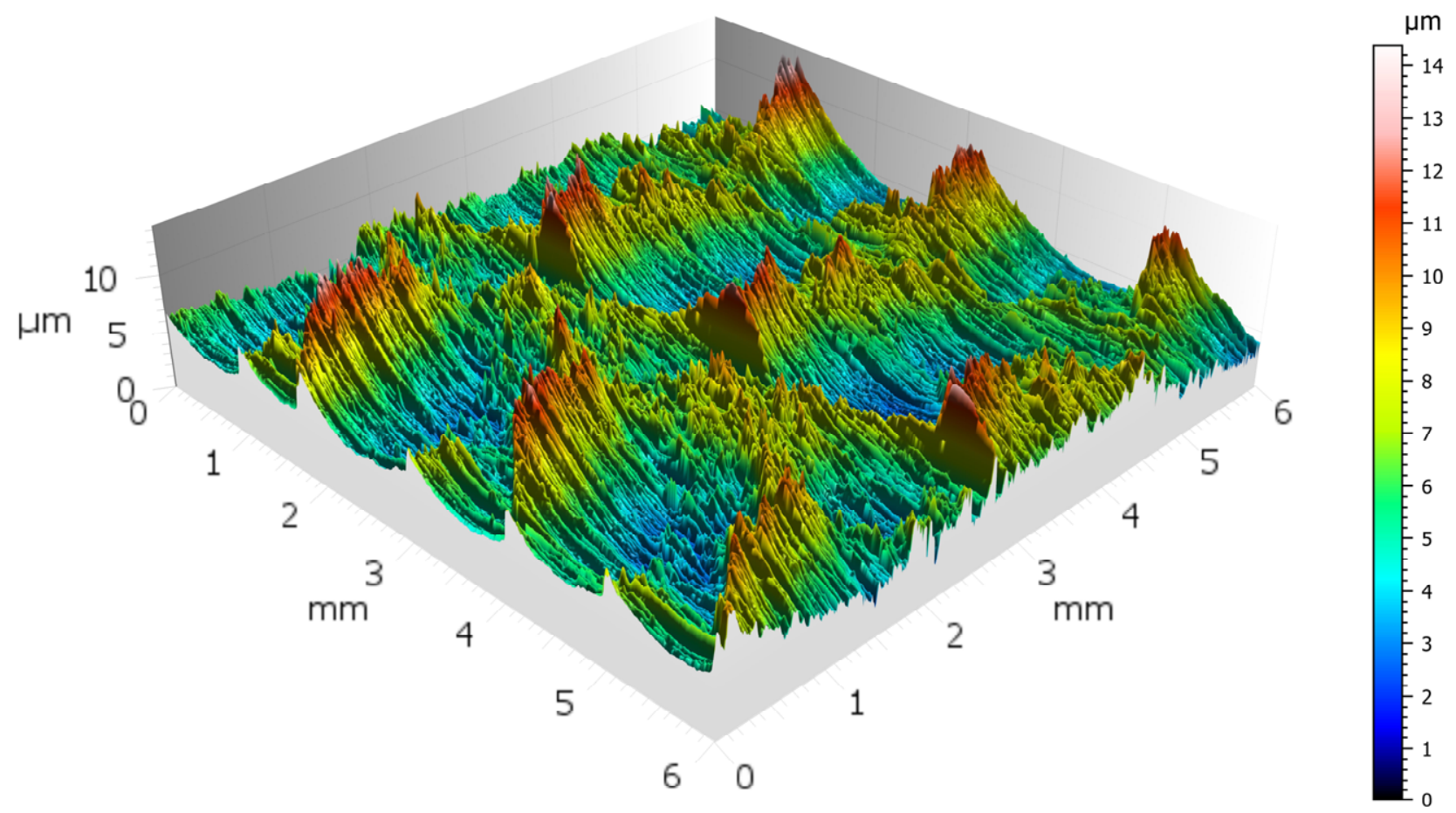

Fig. 3. Topography of the treated surface after friction treatment by the tool with a smooth surface

The histogram of the distribution of the peak heights on the treated surface shows that the largest number of peaks is of average height, which is about $4.5-7 \mu \mathrm{m}$, and their area on a single surface is 1214 grains $/ \mathrm{mm}^{2}$. Small peaks with a height of 3-4.5 $\mu \mathrm{m}$ occupy a small area ranging from $1 \mathrm{grains} / \mathrm{mm}^{2}$ to 4 grains $/ \mathrm{mm}^{2}$ and gradually increase. High peaks, larger than average, with a height of $7 \mu \mathrm{m}$ to $13 \mu \mathrm{m}$ also occupy a sufficient area that is proportional to the area of the peaks of medium height. As the height of the peaks increases, their area decreases significantly from 8 grains $/ \mathrm{mm}^{2}$ to 0.25 grains $/ \mathrm{mm}^{2}$ (Fig. 5). 


\section{Volodymyr Gurey}
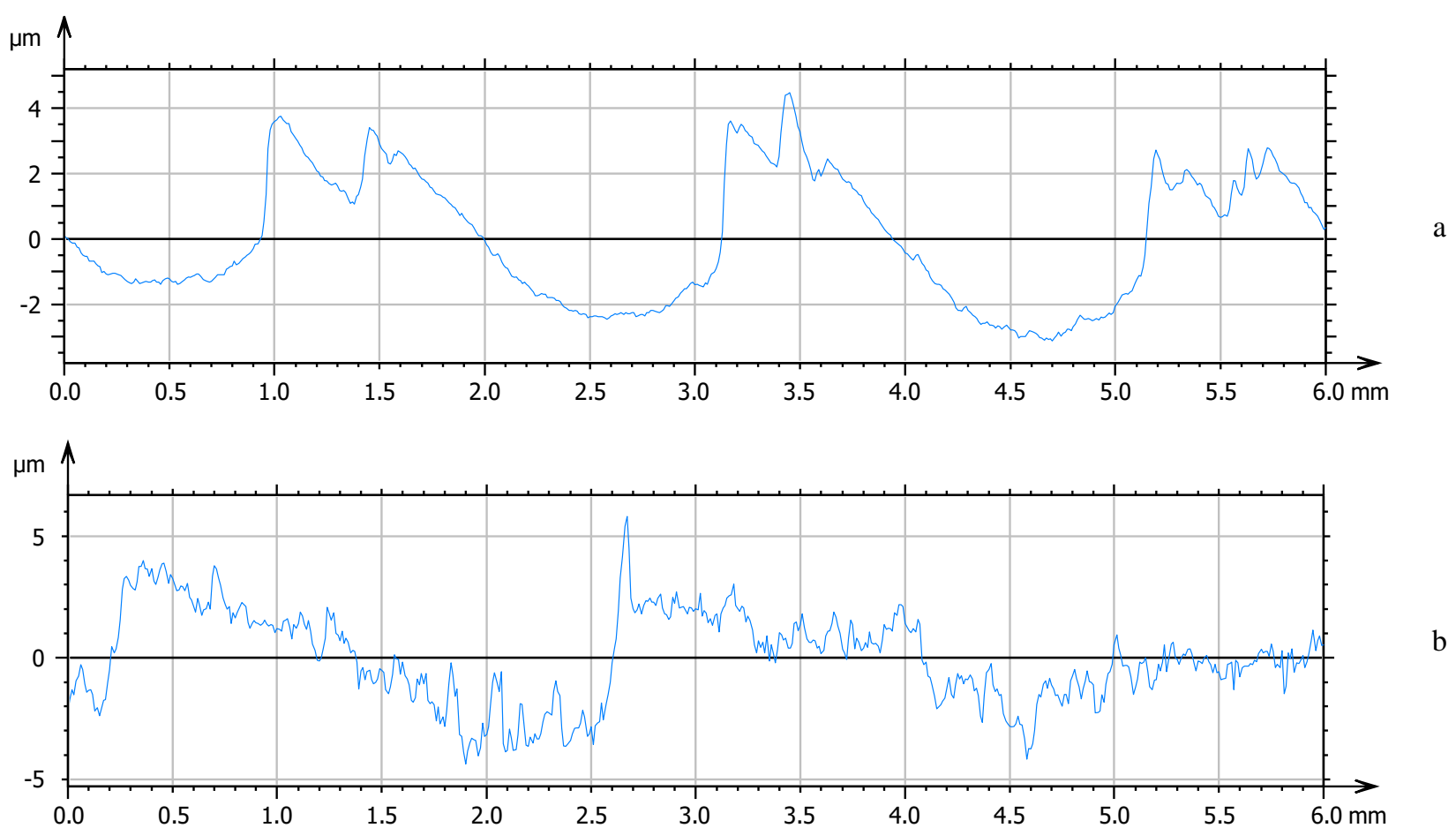

Fig. 4. The waviness of the treated surface in the transverse (a) and longitudinal (b) directions after friction treatment with the tool with a smooth surface

After friction treatment by the tool with transverse grooves on its working part, the topography analysis showed that the peaks on the treated surface are more evenly distributed than after friction treatment by the tool with a smooth surface. The height of the deformed surface is about $10 \mu \mathrm{m}$ (Fig. 6). The surface waviness after friction treatment in the longitudinal and transverse directions is similar to friction treatment by the tool with a smooth working part. In the transverse direction before friction treatment, the height of the waviness is about $6 \mu \mathrm{m}$ and the pitch is about $2 \mathrm{~mm}$, but in the longitudinal direction the waviness is not clearly detected. The height of the profile is also about $5 \mu \mathrm{m}$, the pitch is indefinable (Fig. 7).

grains $/ \mathrm{mm} 2$

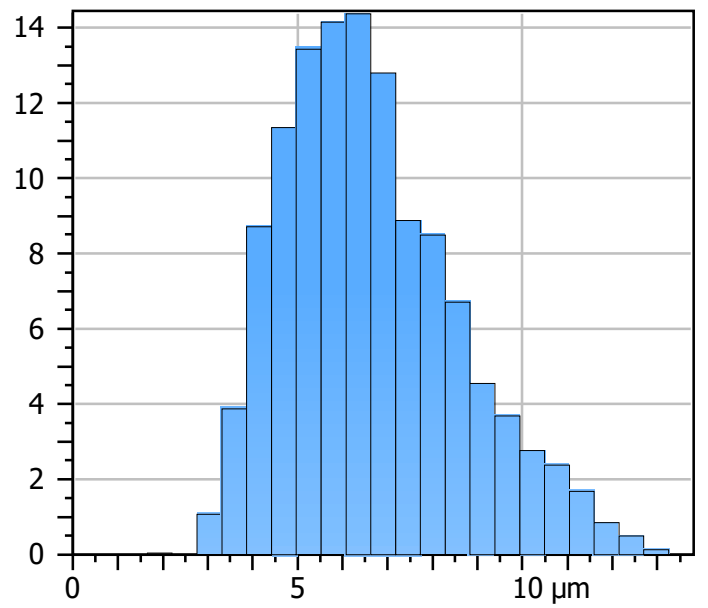

Fig. 5. Peak count histogram of the treated surface after friction treatment by the tool with a smooth surface

Analysis of the histogram of the distribution of the peak heights on the treated surface showed that the largest number of peaks have average height, which is about $5.5-8 \mu \mathrm{m}$, and their area on a single surface is the largest and is about $15-25$ grains $/ \mathrm{mm}^{2}$. Small peaks have height of $4.5-5.5 \mu \mathrm{m}$ and occupy a small area, ranging from 2.5 grains $/ \mathrm{mm}^{2}$ to 7 grains $/ \mathrm{mm}^{2}$ and gradually increase. High peaks, larger than 
average, with a height of $7.5 \mu \mathrm{m}$ to $11.5 \mu \mathrm{m}$ occupy a small area compared to the area of the peaks of medium height. With increasing height of the peaks, their area decreases significantly from 8 grains $/ \mathrm{mm}^{2}$ to 1 grains $/ \mathrm{mm}^{2}$ (Fig. 8). After friction treatment using the tool with cross grooves on its working part the treatment surface has more uniform distribution of peaks with average height. Alternate passage of the smooth part and the groove of the working surface of the tool through the contact area of the tool-part helps to improve the formation the peaks of approximately the same height.

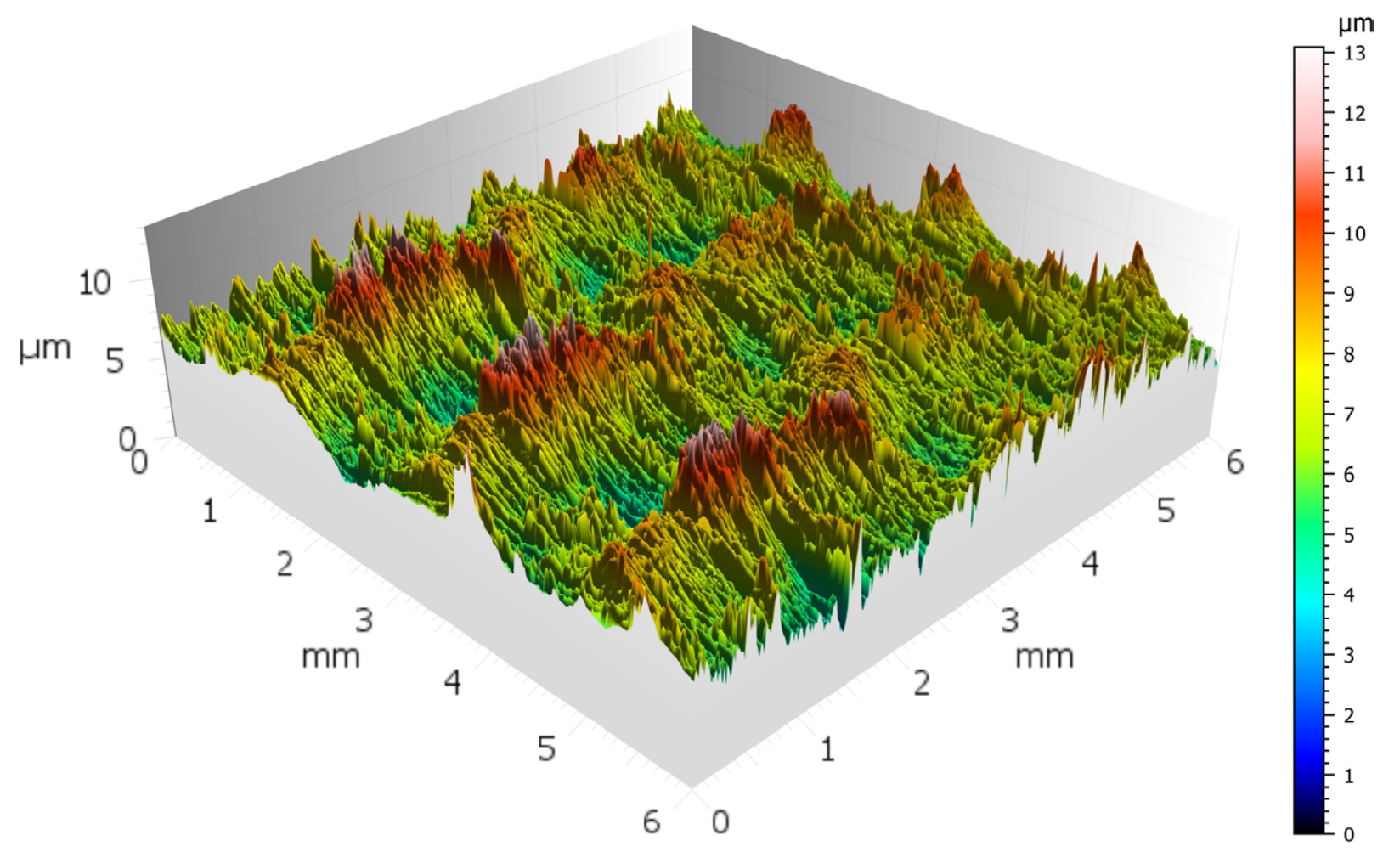

Fig. 6. Topography of the treated surface after friction treatment by the tool with transverse grooves

Stereometric parameters of the studied treated surface are presented as a set of curves that describe the peaks and pits of the profiles and are the non-periodic signals. The average power spectrum density characterizes the frequency of peaks appearance on the studied surface and determines the wavelength that has the largest appearance and its amplitude i.e., the height of the peaks, and describes the state of the treated surface as a whole.

After friction treatment by using the tool with a smooth surface the wavelength power spectrum density is $0.715 \mathrm{~mm}$, and amplitude $-0.2178 \mu \mathrm{m}$. Dominant wavelength is $1.218 \mathrm{~mm}$, maximum amplitude $-0.8962 \mu \mathrm{m}$ (Fig. 9a).

After friction treatment by using the tool with transverse grooves, the wavelength is similar to the previous case and is $0.7512 \mathrm{~mm}$. The amplitude of the spectral wave is much smaller and equal to $0.1375 \mu \mathrm{m}$. The dominant wavelength is slightly smaller than after treatment by using the tool with a smooth working surface and is equal to $1.1179 \mathrm{~mm}$, and the maximum amplitude is almost 2.5 times smaller and equal to $0.3732 \mu \mathrm{m}$ (Fig. 9b).

The spectral density of the distribution of peaks on the investigated surface allows to estimate the frequency of occurrence the certain peaks with a certain identical period. The appearance of peaks with low frequency characterizes the treatment with additional vibrations that occur in this case. Analysing the spectral densities of the distribution of peaks on the treated surfaces, it can be noted that when using the tool with transverse grooves, the area of the spectra is the lowest compared to using the tool with a smooth working part. The peaks of the spectra are similar when used tools with different working parts. Most peaks are detected as a result of using the tool with a smooth working part. 

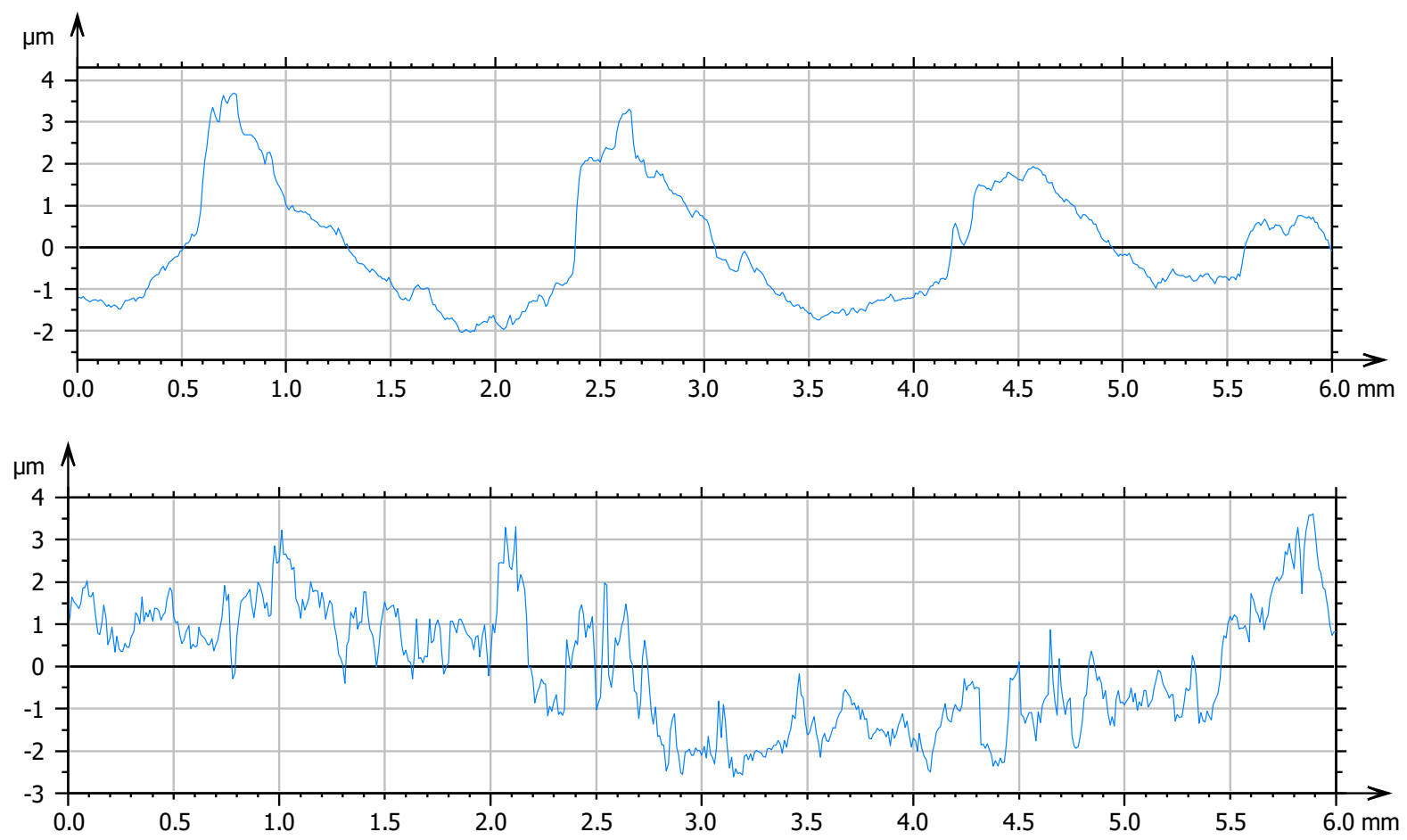

Fig. 7. The waviness of the treated surface in the transverse (a) and longitudinal (b) directions after friction treatment by the tool with transverse grooves

Experimental studies of surface geometry parameters were performed under the same modes of friction treatment by the tools with different working parts. Therefore, the peaks of the spectral density of the distribution of the peaks correspond to the period of passage of the tool through the area of single contact, which in these processing modes was about $1 \mathrm{~mm}$. The intensity of the peaks, which correspond to the period of passage the single contact zone is the smallest, because in this case the interaction of the groove when using with the tool with transverse grooves. When using the tool with a smooth working part, the spectral density of the distribution of the peaks at this stage of treatment is the largest and has several additional peaks.

During friction treatment by using the tool with a smooth working surface, the contact between tool and treated part is constant. The metal of the surface layer in the contact zone is heated to high temperatures close to the melting point. The strength of the surface layer decreases and the value of the coefficient of friction between the tool and the treated surface decreases too. The process of treatment is discontinuous, with some jerks, and it shows into changing in the interaction force components in the contact area of the tool-part during treatment by using the tool with transverse grooves on the working part (see Fig. 2).

An important parameter of the quality of the treated surface is its bearing capacity i.e., the actual surface area along the depth of the distorted (deformed) profile. Bearing capacity determine the percentage that occupied the material of the treated surface, and the void in relation to the total investigated area of the sample. The curve is characterized by three areas: the area of the peaks, which wear out quickly during running-in; the area of medium roughness, which is the basis of the profile and the bearing surface, determines the durability during friction or allowable value of the pair's wear as a whole; area of depressions, the depth of which determines the ability of the surface to retain oil. The bearing capacity curve can be used to estimate how the running-in and wear processes will take place during the friction of the contact surfaces, what is the size of the oil pockets in which the oil can be located, which is very important parameter during the friction processes. 


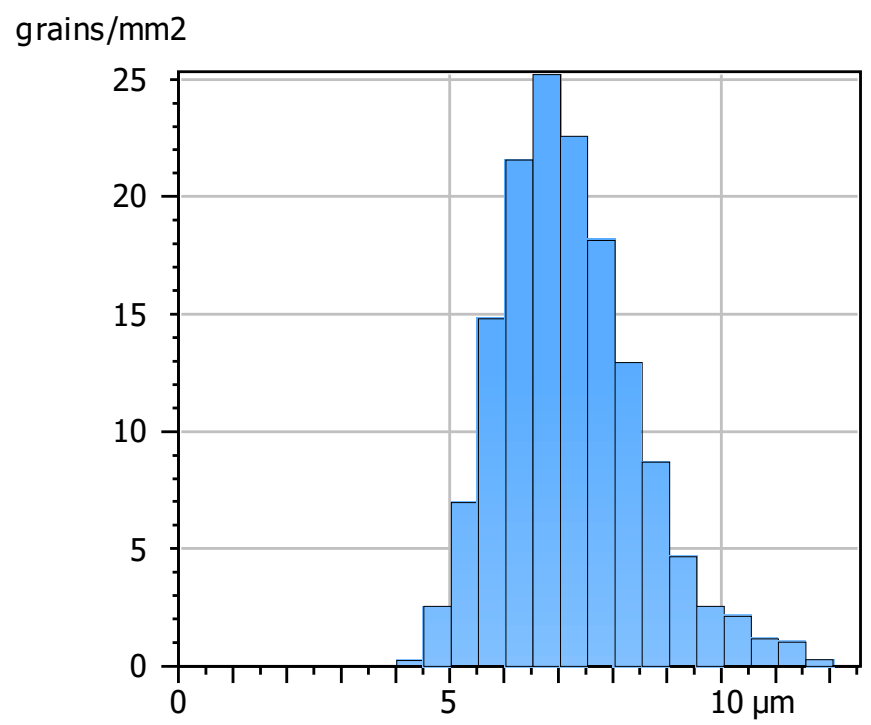

Fig. 8. Peak count histogram of the treated surface after friction treatment by the tool with transverse grooves
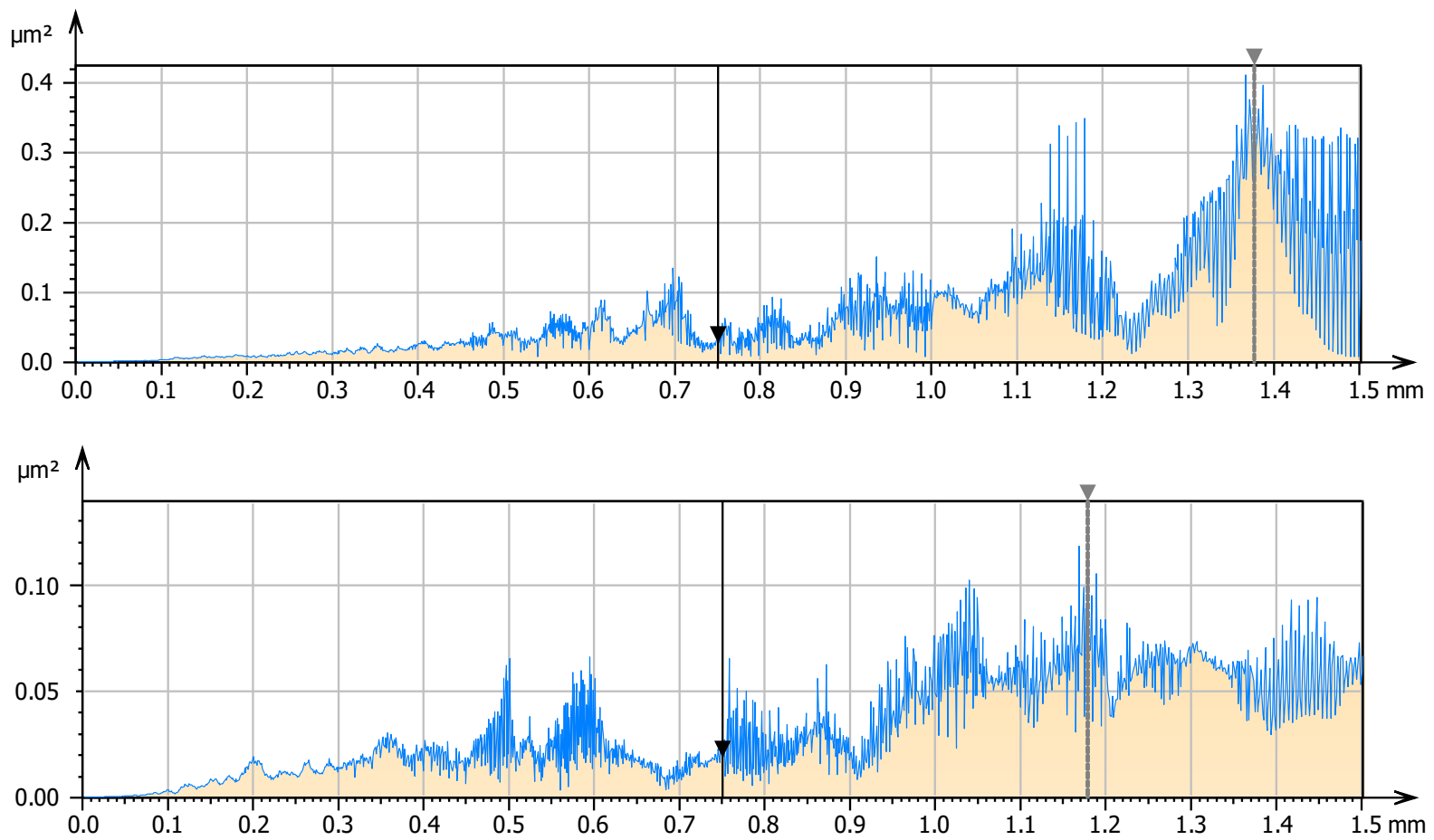

Fig. 9. Averaged power spectrum density after friction treatment by using the tool with a smooth work surface (a) and with transverse grooves (b)

After friction treatment by the tool with a smooth working part, the height of the peaks Spk of the bearing curve is equal 2.773 , the value of the slope of the curve $S k$ is 4.178 , the depth of the depressions $S v k-0.7822$ (Fig. 10). After friction treatment by the tool with transverse grooves, the height of the peaks $S p k$ is equal 1.765, the value of the slope of the curve $S k$ is 2.828 , the depth of the depressions $S v k-$ 0.9821 . Analysing the obtained component parameters of the load-bearing curve of the profiles obtained by surface treatment with tools with different working part, we can say the following: the height of the peaks on the reinforced surface after friction treatment by the tool with grooves on the working part is less than after friction treatment by the tool with a smooth working part, also the angle of the straight section on the bearing curve is smaller and the size of depressions is larger. The area of the peaks affects the time and amount of wear of the surface layer during running-in. The peaks with a lower height promote faster running-in of the contact surfaces of the friction pair. A smaller slope provides a larger area of the actual 


\section{Volodymyr Gurey}

contact surface and reduces the specific load in the friction zone. In this case, the temperatures in the contact zone and the amount of wear intensity decrease, which increases the service life of the friction pair and the allowable value of wear of the contact surfaces. Increasing the depth of the depressions of the profile indicates the possibility of better retention of oil on the contact surface.

The height of macroroughness of the treated surface is the smallest $\left(S_{k}=9.27 \mu \mathrm{m}\right)$ when using the tool with transverse grooves. There are also slight excesses of some peaks of the treated surface over the main surface $\left(S_{p k}=1.72 \mu \mathrm{m}\right)$ and the depth of some pita on the treated surface $\left(S_{v k}=1.74 \mu \mathrm{m}\right)$ compared to the surface treated using the tool with a smooth working surface which has the following surface parameters $-S_{k}=7.24 \mu \mathrm{m}, S_{p k}=2.42 \mu \mathrm{m}, S_{v k}=3.89 \mu \mathrm{m}$.

The nature of the bearing capacity curve of the surface gives information about the possible working of this surface during wear, especially at the initial stage of friction, when the contact surfaces are runningin. When the higher peaks on the contact surface and the smaller their area, the greater specific loads will act on them and the faster will be their crushing and wear. At the initial stage of friction, during running-in, the size of the contact surface will change faster, which is not always desirable for the friction pair. When the larger area occupied by the peaks, preferably of the same height on the treated surface, the less load will act on them and the running-in process will be faster with less loss of size of the surface. Based on this, the treated surface using the tool with transverse grooves has a more favourable surface for wear, which was confirmed by our research on wear resistance $[15,16]$.

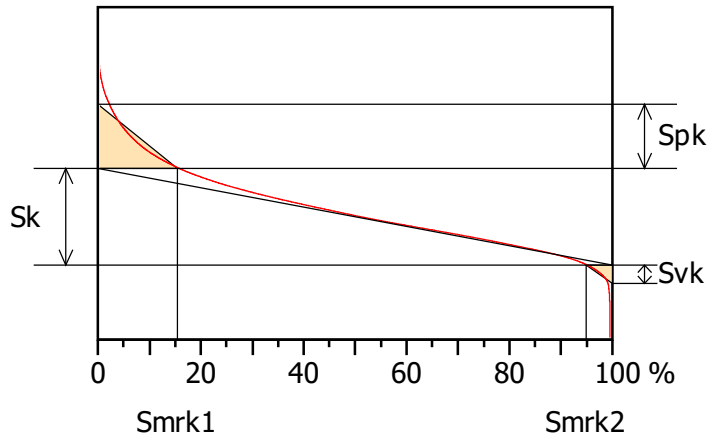

$\begin{array}{ccc} & \text { SS } & \text { TG } \\ \text { Parameters } & \text { Value } & \text { Value } \\ \text { Sk, } \mu \mathrm{m} & 4.178 & 2.828 \\ \text { Spk, } \mu \mathrm{m} & 2.773 & 1.765 \\ \text { Svk, } \mu \mathrm{m} & 0.7822 & 0.9821 \\ \text { Smrk1, \% } & 15.42 & 14.74 \\ \text { Smrk2, \% } & 94.84 & 92.09\end{array}$

Fig. 10. Bearing curve and parameters of the curve of surfaces after friction treatment by the tool with a smooth working part (SS) and by the transverse grooves (TG)

\section{Conclusions}

The interaction force components in the contact area of the tool-part have a wave character with the same phase. The oscillation amplitude of the interaction force components reaches to $0.3-0.4$ of the magnitude of the force that occurs in the contact zone of the tool-part, and the period of oscillations is 20$22 \mathrm{~ms}$. The period of oscillations depends little on the processing modes. Using the tool with transverse grooves leads to decrease the force components of interaction in the contact zone. After friction treatment by using the tool with transverse grooves on its working part on the treated surface more evenly distributed peaks than after friction treatment by using the tool with a smooth surface. Using the tool with transverse grooves on its working part during friction treatment allows to obtain better quality parameters of the treatment surface compared to friction treatment by using the tool with a smooth working part. The treated surface using the tool with transverse grooves has a more favourable surface for wear, which was confirmed by our research on wear resistance.

\section{References}

[1] K. A. Yushchenko, Yu. S. Borisov, V. D. Kuznetsov, and V. M. Korzh, Inzheneriya poverkhni [Surface Engineering]. Kyiv, Ukraine: Naukova dumka Publ., 2007. [in Ukrainian].

[2] A. G. Suslov. Inzheneriya poverhnosti detaley [Surface engineering parts]. Moscow, Russia: Mashinostroenie Publ., 2008. [in Russian].

[3] D. K. Dwivedi, Surface Engineering. Roorkee, India: Springer New Delphi, 2018. 


\section{Topography of the Strengthened Cylindrical Surface after Frictional Continuous Treatment}

[4] P. A. Dearnley, Introduction to Surface Engineering. New-York, USA: Cambridge university press, 2017.

[5] P. Pawlus, R. Reizer, and M. Wieczorowski, "A review of methods of random surface topography modelling", Tribology International, vol. 152, 2020.

[6] P. Pawlus, R. Reizer, M. Wieczorowski, and G. Krolczyk, "Material ratio curve as information on the state of surface topography - A review”, Precision Engineering, vol. 65, pp. 240-258, 2020.

[7] D. G. Waugh, and J. Lawrence, Laser Surface Engineering. Processes and Applications. Sawston, UK: Woodhead Publishing; 2014.

[8] M. A. Montealegre, G. Castro, P. Rey, J. L. Arias, P. Vázquez, and M. González, "Surface treatment by laser technology", Contemporary Materials, vol. I-1, pp.19-30, 2010.

[9] E. L. Papazoglou, A. P. Markopoulos, and D. E. Manolakos, "Experimental research on EDM of AISI O1 tool steel and study of the surface white layer formation”, Procedia Structural Integrity, vol. 10, pp. 235-242, 2018.

[10] Yunhua Xu, Liang Fang, Qihong Cen, and Jinhua Zhu, "Nanostructure and transformation mechanism of white layer for AISI1045 steel during impact wear”, Wear, vol. 258, pp. 537-544, 2005.

[11] L. Tian, "A Short Review on Mechanical Behavior of Nanocrystalline Materials", International Journal of Metallurgy and Metal Physics, vol. 2:008, pp. 2-13, 2017.

[12] V. Gurey, and I. Hurey, "The Effect of the Hardened Nanocrystalline Surface Layer on Durability of Guideways", Lecture Notes in Mechanical Engineering. Advanced Manufacturing Processes. InterPartner 2019, vol. 1, pp. 63-72, 2020.

[13] Geometrical product specifications (GPS) - Surface texture: Areal-Part 600: Metrological characteristics for areal topography measuring methods, ISO 25178-600, 2019.

[14] V. Gurey, and I. Hurey, "Influence of Surface Hardened Nanocrystalline Layers on the Resistance of Contact Fatigue Destruction", Lecture Notes in Mechanical Engineering. Advances in Design, Simulation and Manufacturing III, vol. 1: Manufacturing and Materials Engineering, pp. 483-491, 2020.

[15] I. Hurey, T. Hurey, and V. Gurey, "The effect of nanocrystalline layers on the wear resistance of grey cast iron during friction in an oil-abrasive medium", Tribologia, vol. 6, pp. 37-42, 2018.

[16] I. Hurey, T. Hurey, and V. Gurey, "Wear Resistance of Hardened Nanocrystal-line Structures in the Course of Friction of Steel-Grey Cast Iron Pair in Oil-Abrasive Medium”, Lecture Notes in Mechanical Engineering. Advances in Design, Simulation and Manufacturing II, vol. 1, pp. 572-580, 2019. 